\title{
The effect of regionalization on outcome in pulmonary lobectomy: A Canadian national study
}

\author{
Christian J. Finley, MD, MPH, ${ }^{\mathrm{a}}$ Anna Bendzsak, MD, ${ }^{\mathrm{a}}$ George Tomlinson, $\mathrm{PhD},{ }^{\mathrm{b}}$ Shaf Keshavjee, MD, \\ MSc, ${ }^{a}$ David R. Urbach, MD, MSc, ${ }^{b}$ and Gail E. Darling, MD ${ }^{a}$
}

Objective: To examine the effect of regionalization of thoracic surgery services in Canada by evaluating change over time in hospital volumes of pulmonary lobectomy and its impact on length of stay and in-hospital mortality.

\begin{abstract}
Methods: Data on pulmonary lobectomy between 1999 and 2007 were abstracted from the Canadian Institute for Health Information Discharge Abstract Database. In-hospital mortality was analyzed by logistic regression, and log-transformed length of stay was analyzed by linear regression. Cross-sectional analysis of hospital volume, inhospital mortality, and length of stay was performed, controlling for clustering. Within-hospital changes in annual volume on outcome was analyzed using multivariable logistic regression, controlling for Charlson comorbidity index and other confounders.
\end{abstract}

Results: Of 19,732 patients, 10, $281(52 \%)$ were male, with an average age of 63.3 years. There was a $45 \%$ (95\% confidence interval, $21-61 ; P=.001$ ) relative risk reduction in in-hospital mortality with a $19 \%$ reduction in length of stay $(95 \%$ confidence interval, $12-25 ; P<.0001)$. On comparison of volume between hospitals, an increase of 20 cases was associated with a $15 \%$ relative risk reduction $(95 \%$ confidence interval, $9-19 ; P<.0001)$ in in-hospital mortality and a $5 \%$ relative decrease $(95 \%$ confidence interval, $3-7 ; P<.001)$ in length of stay. Within hospitals there was a nonsignificant relationship between volume and in-hospital mortality.

Conclusions: In-hospital mortality and length of stay for lobectomies have decreased in Canada. In multivariate analysis, volume was associated with improved in-hospital mortality, but there was no reduction in mortality when volume was increased within a given hospital. However, the proportion of patients treated in highvolume centers has increased over time, inferring the importance of high-volume centers in improved outcomes. This supports regionalization policies for pulmonary lobectomy. (J Thorac Cardiovasc Surg 2010;140:757-63)

Earn CME credits at

http://cme.ctsnetjournals.org

The association between hospital volumes and surgical outcomes has been well documented for many high-risk surgical procedures, including surgery for lung cancer. ${ }^{1-4}$ In the United States, a large body of evidence suggests a consistent relationship between larger hospital volumes and improved short-term outcomes for lobectomy and pneumonectomy. ${ }^{4-6}$ Improvements in long-term outcomes are also related to institutional volume. ${ }^{4}$ There is also a suggestion that institu-

\footnotetext{
From the Division of Thoracic Surgery ${ }^{\mathrm{a}}$ and Division of Clinical Decision Making and Health Care, ${ }^{\mathrm{b}}$ Toronto General Hospital, Toronto, Ontario, Canada.

Disclosures: None.

Read at the 90th Annual Meeting of The American Association for Thoracic Surgery,

Toronto, Ontario, Canada, May 1-5, 2010.

Received for publication April 30, 2010; revisions received June 19, 2010; accepted for publication June 28, 2010.

Address for reprints: Christian J. Finley, MD, MPH, Toronto General Hospital,

Division of Thoracic Surgery, 200 Elizabeth St, EN9-946 Toronto, ON, M5G

2C4, Canada (E-mail: christian.finley@uhn.on.ca).

$0022-5223 / \$ 36.00$

Copyright (C) 2010 by The American Association for Thoracic Surgery

doi:10.1016/j.jtcvs.2010.06.040
}

tional designation as a cancer center provides improved outcomes beyond volume. ${ }^{7}$ The evidence for this volumeoutcome relationship is somewhat controversial, however, because there are several studies from the United Kingdom, Canada, and the United States that fail to show any benefit to hospital volume on outcome in pulmonary resections. ${ }^{8-10}$ Furthermore, many smaller institutions are considered to deliver high-quality care and have among the most favorable outcomes reported in the literature. ${ }^{11}$

There is no universally accepted volume standard for a large-volume center, with many publications quoting differing thresholds. The Society of Thoracic Surgeons database, ${ }^{12}$ a voluntary dedicated thoracic database, reports a median of 31.4 lobectomies per participant per year, but numbers range from 1 to 128 . Hospital lobectomy volumes ranging from 19 to 37 per year have been considered large volume, ${ }^{1,13}$ and in Canada, Ontario has set a standard of 150 anatomic lung resections per year as a target for high-volume centers. ${ }^{14}$ In searching for a volume threshold above which outcomes are improved, factors other than volume are often found to have more influence on the variability in outcomes. ${ }^{15}$

The evidence for a positive relationship between hospital volumes and surgical outcomes has led to the suggestion that care be regionalized primarily in large-volume centers. ${ }^{16}$ In Canada, thoracic surgery services have been formally 


$$
\begin{aligned}
& \text { Abbreviations and Acronyms } \\
& \text { CI }=\text { confidence interval } \\
& \text { ICD }=\text { International Classification of Diseases } \\
& \text { IHM }=\text { in-hospital mortality } \\
& \text { LOS }=\text { length of stay } \\
& \text { SD }=\text { standard deviation }
\end{aligned}
$$

regionalized in British Columbia and Ontario as a result of this literature with added funding and resources being added to facilitate optimum care in 2001 and 2004, respectively. ${ }^{14,17}$ In the largest province of Ontario, the regionalization came with necessary requirements for facilities and availability of specialized care. Informally, many other provinces have amalgamated existing smaller centers to effectively regionalize their services without explicit added resources. Several authors caution about potential untoward side effects of regionalization policies, ${ }^{11}$ and as yet, the effect of manipulating hospital volumes on surgical outcomes for patients has not been established. Authors theorize that patient outcomes will be improved by increasing an institution's volume or alternatively by referring patients to existing high-volume centers.

To address the effect of regionalization on outcomes in thoracic surgery, pulmonary lobectomy was chosen because it is the most commonly performed major thoracic oncologic surgical procedure with a reported mortality ranging from $0.8 \%$ to $3.8 \%{ }^{9,12,18,19}$ and a morbidity rate of $15.3 \%$ to $47 \% .^{12,18,19}$

The objective of this study was to examine changes in length of stay (LOS) and in-hospital mortality (IHM) after pulmonary lobectomy in Canada over time and to describe relationships between these short-term surgical outcomes and changes in hospital volumes, both between and within hospitals.

\section{MATERIALS AND METHODS}

All pulmonary lobectomies for patients age 18 years and older recorded in the Canadian Institute for Health Information Discharge Abstract Database from 1999 to 2007 were included (International Classification of Diseases [ICD], 9th Revision code 44.4 for cases up to 2004, and 10th revision code 1.GR.89.DA/NW/QB). Extended resections and bilobectomy were excluded. The Discharge Abstract Database contains all hospital discharges in Canada with the exception of those performed in Quebec and Manitoba outside Winnipeg. De-identified information on age, gender, admission date, LOS, IHM, province of operation, institution number, surgeon, and patient disposition were collected. To adjust for patient risk factors, the Charlson comorbidity index was calculated using pre-hospitalization ICD-9 and 10 diagnosis codes, ${ }^{20}$ a method that has been validated. ${ }^{21,22}$ The study was approved by the institutional research ethics board at the University Health Network.

Descriptive analyses were conducted to determine the change in number of cases performed, IHM, and LOS over time. Random effects logistic regression was used for the analysis of IHM, and random effects linear regression was used for the log-transformed LOS analysis. Unadjusted regression was performed to examine trends in outcomes over time. The effect of an- nual hospital lobectomy volumes on outcomes was examined by modeling yearly hospital volume by IHM and LOS, adjusting for calendar year, gender, age, Charlson comorbidity index (each score analyzed separately), province of care (using Ontario as the reference province), and the average volume per hospital over the study period. An institutional increase in volume of 20 cases per year was considered clinically relevant.

We then examined the effect of changing lobectomy volumes within an individual hospital. Both absolute yearly differences from the overall mean per hospital and relative yearly differences from the overall mean per hospital were examined in multivariate models and yielded similar results. We present the results from the analysis using absolute differences in yearly hospital volumes. An institutional increase in volume of 20 cases per year was considered clinically relevant.

In the multivariate model, volume was analyzed as a continuous variable. To present groupings of hospitals into low, medium, and high volume, the number of cases performed over time was examined using different thresholds for "high volume," ranging from 50 to 100 cases per year, and "medium volume", as 10 cases per year up to the threshold of "high volume." After plotting the results graphically, a threshold of 60 cases per year (or $\sim 1$ per week) was determined to be clinically relevant as the cutoff for "high volume" because our data supported changes in time in institutional numbers at this value. Low volume was defined as fewer than 10 cases per year or approximately 1 per month.

\section{RESULTS}

The study interval from 1999 to 2007 included 19,732 patients, of whom 10,281 (52\%) were male. The average age was 63.3 (standard deviation $[\mathrm{SD}]=13.2$; range, 18-94) years. The average Charlson comorbidity index was 2.26 $(\mathrm{SD}=1.30)$. The average Charlson comorbidity index increased over the study interval from 1.9 to 2.3 . The number of procedures performed per year increased over the study period from 2120 to 2561, as shown in Figure 1. Preoperatively, $82.8 \%$ of patients had a diagnosis of cancer. A decrease in lobectomies in 2003 is thought to be attributable to the severe acute respiratory syndrome outbreak.

IHM per year is shown in Figure 2. The unadjusted analysis revealed a $45 \%$ relative risk reduction $(95 \%$ confidence interval $[\mathrm{CI}], 21-61 ; P=.001)$ in IHM over the study period and a $19 \%$ reduction in $\operatorname{LOS}(95 \% \mathrm{CI}, 12-25 ; P<.0001)$. The IHM yearly rate decreased over the study period from a peak of $3.1 \%$ in 1999 to $1.95 \%$ in 2007 . Similarly, LOS yearly rates decreased from 10.4 days $(\mathrm{SD}=12.2)$ in 1999 to 8.9 days $(\mathrm{SD}=10.1)$ in 2007.

When we examined the effect of volume on mortality, we found that hospitals performing fewer than 10 lobectomies per year had the highest mortality at $4.8 \%$, whereas those performing more than 100 lobectomies per year had the lowest mortality at $1.8 \%$ (Figure 3 ).

The risk-adjusted models for IHM and LOS are shown in Table 1. Both lower IHM and lower LOS were associated with institutions with higher volumes. After adjusting for age, gender, and Charlson index, there was a $15 \%$ relative risk reduction $(95 \% \mathrm{CI}, 9-19 ; P<.0001)$ for IHM and a $5 \%$ relative decrease $(95 \% \mathrm{CI}, 3-7 ; P<.001)$ in LOS for every 20 additional cases performed per hospital. Table 1 shows that male gender and increasing age are 


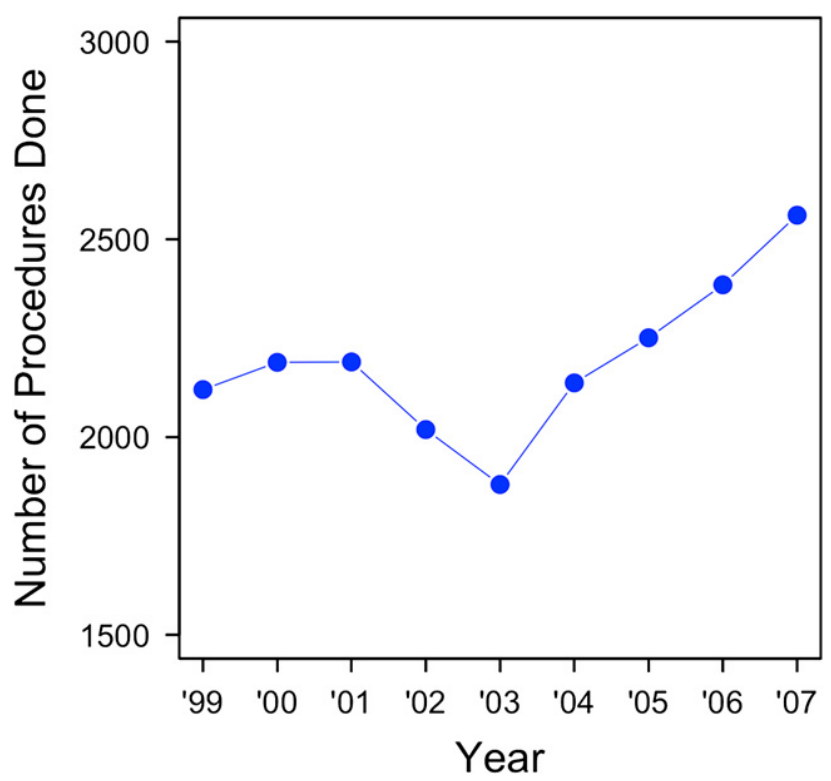

FIGURE 1. Number of pulmonary lobectomies in Canada per year. *Error bars represent $95 \%$ CIs.

associated with worse outcomes. However, after adjusting for known patient factors and volume, year of procedure remains a significant factor.

When examining changes in volume that occurred within an individual hospital, there was a nonsignificant relationship between increasing volumes within hospitals and IHM, with a $-5 \%(95 \% \mathrm{CI}, 6$ to $-18, P=.39)$ relative decrease in mortality for each additional 20 cases performed within a given hospital. In contrast, there was a significant

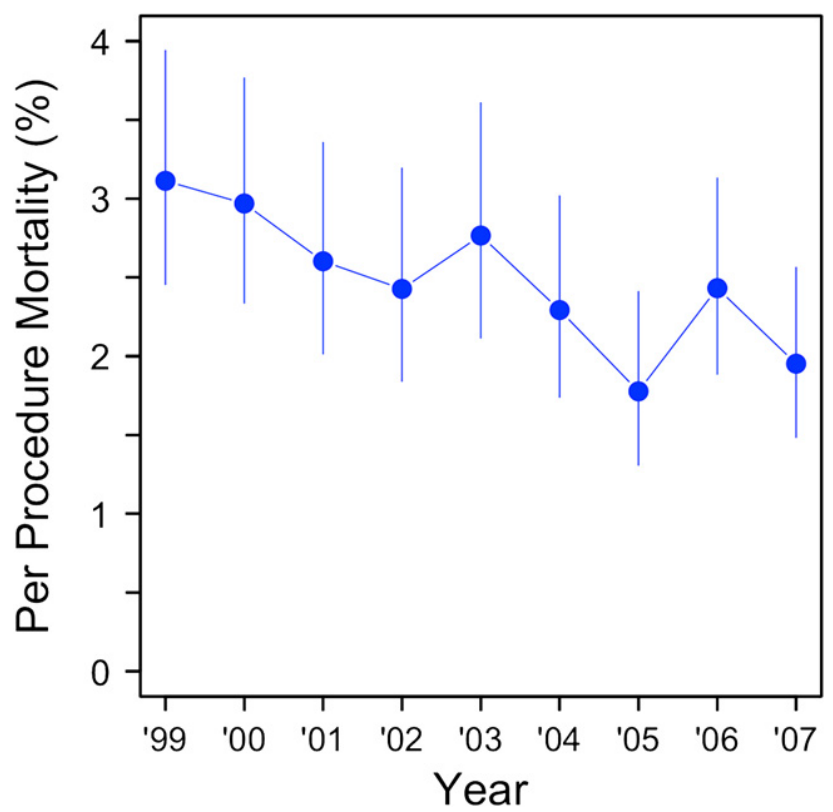

FIGURE 2. Pulmonary lobectomy mortality rate in Canada per year. *Error bars represent $95 \%$ CIs.

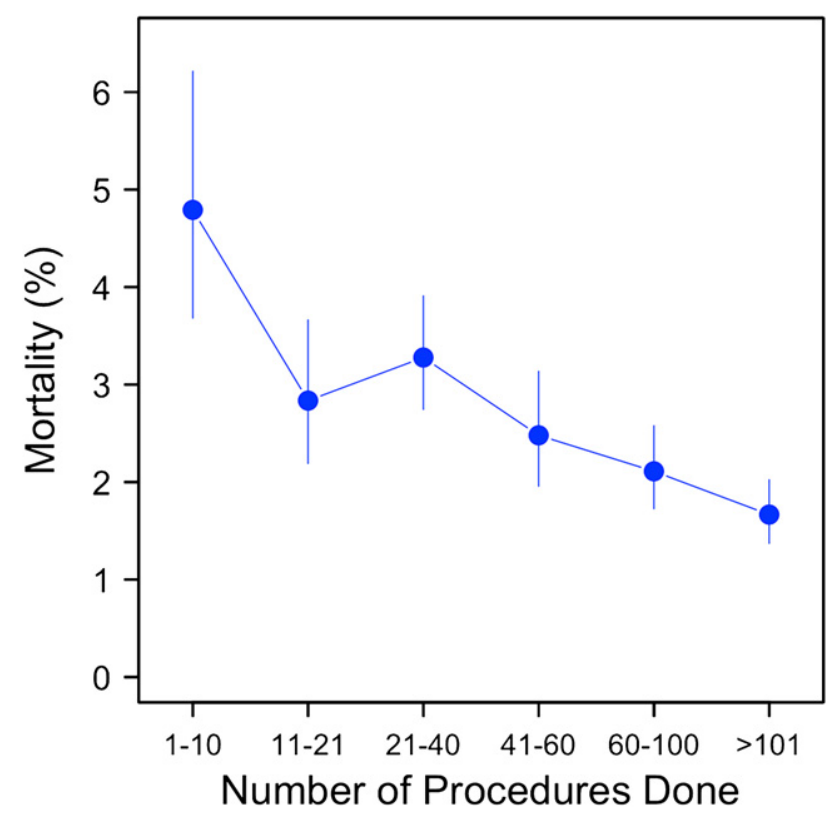

FIGURE 3. Mortality as it relates to the number of procedures done crosssectionally over the entire study.

decrease in LOS, with a $4 \%$ relative decrease in LOS $(95 \%$ CI, $1-6 ; P=.0005)$ for each incremental increase in volume of 20 cases within a given hospital. There was a significant difference in LOS between the provinces, but not for IHM (data not shown).

The change in percentage of patients treated in centers with different volumes over the study period is shown in Figure 4. In 1999, 77 institutions performed lobectomies in Canada, compared with 69 institutions in 2007. The percentage of cases performed in "high-volume" centers ( $>60$ cases per year) increased over time from $49.7 \%$ to $65 \%$, the percentage of cases performed in "medium-volume" centers (11-60 cases per year) decreased from $43.4 \%$ to $31 \%$, and the percentage of cases performed in small-volume centers decreased from $6.9 \%$ to $3.2 \%$, suggesting that cases overall are being increasingly performed in high-volume centers.

\section{DISCUSSION}

This study presents the relationship between hospital volumes and short-term outcomes for pulmonary lobectomy in Canada during the study interval of 1999 to 2007 . We found a significant decrease in IHM and LOS despite increased numbers of lobectomies performed in patients with increased comorbidities. For the approximately 2600 cases of lung lobectomies performed in 2007 in Canada, this change results in approximately 26 lives saved and 3900 patient days in hospital avoided. It is a considerable improvement in the delivery of one thoracic operation, which could have even more profound impact when extrapolated across other thoracic surgical procedures. 
TABLE 1. Multivariable regression model of in-hospital mortality and length of stay

\begin{tabular}{|c|c|c|c|c|}
\hline In-hospital mortality & Odds ratios & Lower 95\% & Upper $95 \%$ & $P$ value \\
\hline $\begin{array}{l}\text { Yearly mean } \\
\text { volume (per patient) }\end{array}$ & 0.992 & 0.989 & 0.995 & $<.0001$ \\
\hline Relative volume & 1.003 & 0.997 & 1.008 & .3899 \\
\hline Calendar year & 0.952 & 0.916 & 0.989 & .0107 \\
\hline Gender (male) & 1.753 & 1.435 & 2.142 & $<.0001$ \\
\hline Age (per 10 y) & 1.628 & 1.482 & 1.788 & $<.0001$ \\
\hline Charlson $=1$ & 0.396 & 0.227 & 0.694 & .0009 \\
\hline Charlson $=2$ & 0.27 & 0.205 & 0.356 & $<.0001$ \\
\hline Charlson $=3$ & 0.402 & 0.292 & 0.555 & $<.0001$ \\
\hline Charlson $=4$ & 1.371 & 0.832 & 2.258 & .2064 \\
\hline Charlson $=5$ & 0.258 & 0.169 & 0.393 & $<.0001$ \\
\hline Charlson $=6+$ & 1.009 & 0.535 & 1.9 & .9785 \\
\hline Length of stay & Odds ratios & Lower 95\% & Upper $95 \%$ & $P$ value \\
\hline $\begin{array}{l}\text { Yearly mean } \\
\text { volume (per patient) }\end{array}$ & 0.997 & 0.996 & 0.999 & $<.0001$ \\
\hline Relative volume & 0.998 & 0.997 & 0.999 & .0005 \\
\hline Calendar year & 0.982 & 0.979 & 0.986 & $<.0001$ \\
\hline Gender (male) & 1.033 & 1.016 & 1.05 & .0001 \\
\hline Age (per 10 y) & 1.098 & 1.09 & 1.105 & $<.0001$ \\
\hline Charlson $=1$ & 1.087 & 1.039 & 1.137 & .0001 \\
\hline Charlson $=2$ & 1.003 & 0.974 & 1.034 & .4097 \\
\hline Charlson $=3$ & 0.998 & 0.964 & 1.033 & .4436 \\
\hline Charlson $=4$ & 1.369 & 1.26 & 1.487 & $<.0001$ \\
\hline Charlson $=5$ & 1.037 & 0.998 & 1.078 & .0297 \\
\hline Charlson $=6+$ & 1.39 & 1.273 & 1.518 & $<.0001$ \\
\hline
\end{tabular}

The multivariate model shows that although mortality decreases with increasing volume when comparing between hospitals, there is no effect on mortality when individual hospitals increase their volumes. Furthermore, we observed both a decrease in overall adjusted mortality over time and an increase in volumes in those hospitals performing highvolume procedures over time, similar to US reports. ${ }^{23,24}$ Although the total volume of cases increased over time could explain this, only $5.6 \%$ of institutions crossed from the medium-volume institutions to high-volume institutions. Thus, it is likely that the decrease in mortality is associated with a larger proportion of lobectomies being delivered in high-volume centers and the cessation of the lowervolume institutions with their associated average higher mortality. This suggests that for lobectomy in Canada, the volume-outcome relationship is best optimized by selective referral to existing large-volume institutions.

Factors affecting mortality include patient factors and the average procedural volume of the hospital where the lobectomy is performed. Patient and volume characteristics seem to be changing over time with overall increasing Charlson comorbidity index and proportion of patients being treated in high-volume centers, whereas age is relatively stable. However, mortality is improving over time beyond that explained by these factors. Whether this added effect reflects improved staging, surgical selection, or early interventions on critically ill patients ${ }^{25}$ is unclear. The increasing availability of video-assisted lobectomy offers a potential surgical factor that may contribute to improved outcomes in larger institutions, but the adoption of this technique in Canada is still ongoing, and with the limitations of the coding, its effect is inaccessible. A shift in procedures to high-volume hospitals is the sole potentially externally modifiable variable. Indeed, there has clearly been a movement of patients to higher-volume centers.

Our study also addresses the question of what occurs to outcomes when the volumes in individual centers change. Notably, increasing an individual center's volume failed to show any change in mortality, but a significant improvement in LOS was observed when individual hospital volumes increased. LOS is frequently used as a marker of quality of care, with reduced LOS representing a surrogate marker for improved management of patients, including preoperative patient selection, operative care, postoperative course, and reduced complications. Its improvement with increased hospital volumes may represent change in practice over time or improved efficiency of care in response to healthcare economic pressures.

\section{Study Limitations}

Our study has several limitations. Accounting for casemix and comorbidity is required in volume-outcome 


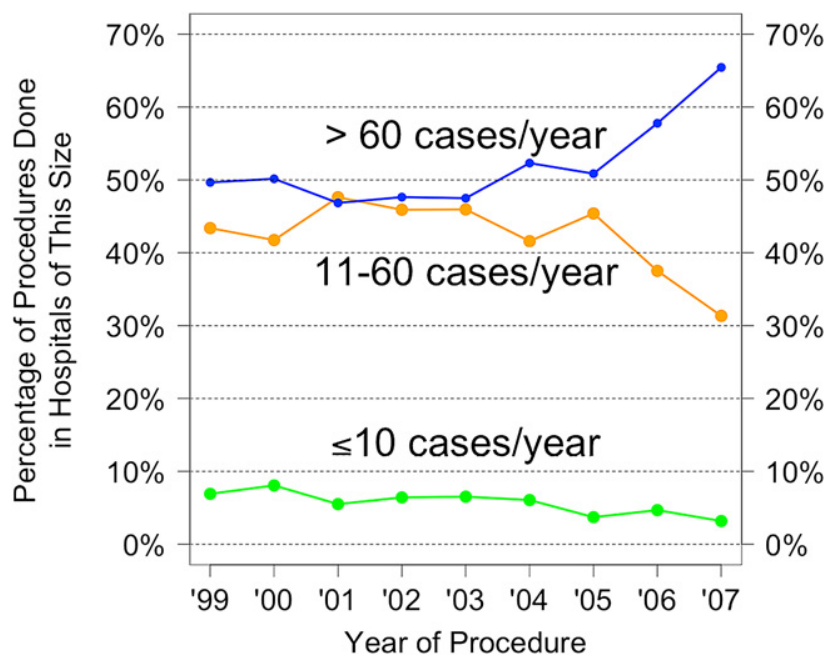

FIGURE 4. Changing percentage of patients treated at centers with different volumes.

relationship investigations, but we were limited to the use of the Charlson comorbidity index, which has been validated for population-based studies and used elsewhere in volume-outcome research. Its use, however, of comorbid illnesses, such as congestive heart failure, diabetes, human immunodeficiency virus, renal failure, and cancer, does not include all accepted factors that increase the risk of pulmonary resection. Other important markers, such as forced expiratory volume in 1 second, carbon monoxide diffusion in the lung, and exercise testing, are not available in the Canadian Institute for Health Information database and are not part of the Charlson index. In our study, IHM and LOS did not change uniformly with progressive Charlson comorbidity scores as would be expected. This could be explained by the expected worse outcomes in patients undergoing anatomic lung resection for inflammatory reasons, many of whom would have a correspondingly lower Charlson score. As such, each score was analyzed separately and not linearly to account for this effect. Furthermore, although the Charlson index accounts for malignancy, we do not have stage information in our database, which limits our ability to control for patients with more advanced disease, although arguably this should not affect short-term outcome in well-selected patients.

There was a coding change from ICD-9 to ICD-10 over the course of our study, which was incorporated at different times in each province. The ICD-10 coding system is more detailed than the ICD-9 system, and thus it is possible that the same spectrum of cases was not captured by our use of the ICD-10 codes as was captured for the use of ICD-9 codes. Because the results were consistent across the study period, however, this is likely not a significant factor. The Charlson comorbidity index is formed from ICD coding, but it has been shown that the change in coding did not affect its performance in capturing comorbidity. ${ }^{20-22}$
There has been active promotion of the concept of regionalization of thoracic surgery services to high-volume centers based on the quantity of cross-sectional evidence for volume-outcome relationships. Our results support the crosssectional relationship between short-term outcomes and higher-volume centers. In addition, we show that, over time, there has been an improvement in short-term mortality, and this change is in part due to more cases being done in higher-volume centers rather than within-hospital improvement. These results support the idea that services should continue to be regionalized to high-volume centers. However, regionalization is clearly more than just volume and includes other processes of care that we have not measured, such as specialized services, staff, and experience. This study suggests that systematic initiatives in regionalization of thoracic surgery are moving us forward in improving outcomes of patients undergoing lobectomy. It also underscores the importance of outcome data collection and analysis going forward to accurately assess the efficacy of our attempts to improve the quality and efficiency of health care delivery.

\section{CONCLUSIONS}

During a period when several initiatives were implemented to improve patient care in thoracic surgery in Canada, IHM and LOS for pulmonary lobectomies decreased. High-volume centers demonstrated significantly improved overall outcomes. Although increased volume within a hospital was related to decreasing LOS, we did not find a significant relationship between mortality and changing yearly volume within a given hospital. The proportion of patients treated in high-volume centers has increased over time, and volume is associated with improved outcome in the multivariate model. These results infer an important role of high-volume centers in improved LOS and IHM. Our results support the policy that services should continue to be regionalized to high-volume centers. They also underscore the importance of prospective outcome data analysis to accurately assess the efficacy of efforts to improve quality and efficiency of health care delivery.

\section{References}

1. Birkmeyer J, Therese S, Siewers A, Goodney P, Wennberg D, Lucas L. Surgeon volume and operative mortality in the United States. N Engl J Med. 2003;349: 2117-27.

2. Begg C, Cramer L, Hoskins W, Brennan M. Impact of hospital volume on operative mortality for major cancer surgery. JAMA. 1998;280:1747-51.

3. Gordon TA, Burleyson GP, Tielsch JM, Cameron JL. The effects of regionalization on cost and outcome for one general high-risk surgical procedure. Ann Surg. 1995;221:43-9.

4. Bach PB, Cramer LD, Schrag D, Downey RJ, Gelfand SE, Begg CB. The influence of hospital volume on survival after resection for lung cancer. $N$ Engl J Med. 2001;345:181-8.

5. Hannan EL, Radzyner M, Rubin D, Dougherty J, Brennan MF. The influence of hospital and surgeon volume on in-hospital mortality for colectomy, gastrectomy, and lung lobectomy in patients with cancer. Surgery. 2002;131:6-15.

6. Finlayson E, Goodney P, Birkmeyer J. Hospital volume and operative mortality in cancer surgery: a national study. Arch Surg. 2003;138:721-6. 
7. Birkmeyer NJ, Goodney PP, Stukel TA, Hillner BE, Birkmeyer JD. Do cancer centers designated by the National Cancer Institute have better surgical outcomes? Cancer. 2005; 103:435-41.

8. Simunovic M, Rempel E, Thériault ME, Coates A, Whelan T, Holowaty E, et al. Influence of hospital characteristics on operative death and survival of patients after major cancer surgery in Ontario. Can J Surg. 2006;49:251-8.

9. Treasure T, Utley M, Bailey A. Assessment of whether in-hospital mortality for lobectomy is a useful standard for the quality of lung cancer surgery: retrospective study. BMJ. 2003;327:73.

10. Khuri SF, Daley J, Henderson W, Hur K, Hossain M, Soybel D, et al. Relation of surgical volume to outcome in eight common operations: results from the VA National Surgical Quality Improvement Program. Ann Surg. 1999;230:414-32.

11. Urschel JD, Urschel DM. The hospital volume-outcome relationship in general thoracic surgery. Is the surgeon the critical determinant? J Cardiovasc Surg. 2000;41:153-5

12. Boffa DJ, Allen MS, Grab JD, Gaissert HA, Harpole DH. Wright CD Data from The Society of Thoracic Surgeons General Thoracic Surgery database: the surgical management of primary lung tumors. J Thorac Cardiovasc Surg. 2008;135:247-54.

13. Finlayson EV, Birkmeyer JD. Effects of hospital volume on life expectancy after selected cancer operations in older adults: a decision analysis. J Am Coll Surg. 2003;196:410-7.

14. Available at: www.cancercare.on.ca/cms/one.aspx?pageId $=41173$.

15. Meguid R, Weiss E, Chang D, Brock M, Yang S. The effect of volume on esophageal cancer resections: what constitutes acceptable resection volumes for centers of excellence? J Thorac Cardiovasc Surg. 2009;137:23-9.

16. Birkmeyer JD. Should we regionalize major surgery? Potential benefits and policy considerations. J Am Coll Surg. 2000;190:341-9.

17. BC Thoracic Surgery. About Us. Vancouver, BC: 2006; BC Thoracic Surgery. Available at: http://www.thoracicsurgery.bc.ca/aboutus.html. Accessed March 9, 2009.

18. Berry M, Hanna J, Tong B, Burfeind W, Harpole D, D'Amico T, et al. Risk factors for morbidity after lobectomy for lung cancer in elderly patients. Ann Thorac Surg. 2009;88:1093-9.

19. Meguid R, Brooke B, Chang D, Sherwood T, Brock M, Yang S. Are surgical outcomes for lung cancer resections improved at teaching hospitals? Ann Thorac Surg. 2008;85:1015-25.

20. Li B, Evans D, Faris P, Dean S, Quan H. Risk adjustment performance of Charlson and Elixhauser comorbidities in ICD-9 and ICD-10 administrative databases. BMC Health Serv Res. 2008;8:12.

21. Iezzoni LI. Risk Adjustment for Measuring Health Care Outcomes. Chicago, IL: Health Administration Press; 2003.

22. Quan H, Sundararajan V, Halfon P, Fong A, Burnand B, Luthi JC, et al. Coding algorithms for defining comorbidities in ICD-9-CM and ICD-10 administrative data. Med Care. 2005;43:1130-9.

23. Memtsoudis SG, Besculides MC, Zellos L, Patil N, Rogers SO. Trends in lung surgery: United States 1988 to 2002. Chest. 2006;130:1462-70.

24. Ho V, Heslin MJ, Yun H, Howard L. Trends in hospital and surgeon volume and operative mortality for cancer surgery. Ann Surg Oncol. 2006;13:851-8.

25. Ghaferi A, Birkmeyer J, Dimick J. Variation in hospital mortality associated with inpatient surgery. N Engl J Med. 2009;361:1368-75.

\section{Discussion}

Dr Yolonda Colson (Boston, Mass). You are to be commended for your analysis of IHM and LOS in approximately 20,000 patients undergoing lobectomy in Canada during a time of transition to regionalization of thoracic surgical services. Your study demonstrates an impressive $45 \%$ relative risk reduction in IHM and a 19\% reduction in LOS that was associated with an increased number of cases being performed at large-volume centers, alluding to the benefits of regionalization. Although an increase in the number of cases within a hospital did decrease LOS, it did not lead to a decrease in mortality, suggesting that an increase in volume alone is not the sole answer.

In an attempt to better understand these observations and their applicability to other scenarios and health care systems, I have 3 questions based on your presentation and article.
First, would you please describe how regionalization was initiated and carried out in Canada, because we are not as familiar with that, and how it relates to the data that you presented? For example, was the transition gradual or does a conscious mandated shift of patients to large-volume centers account for the abrupt increase in the percentage of cases done at high-volume centers that starts in about 2005 as demonstrated in your article, and can you comment on the driving force behind the marked increase in the number of lobectomies that you noted starting in about 2003 ?

Dr Finley. Canada delivers health care on a provincial basis, and so the regionalization process occurred in differing ways and different scenarios in each province. The first to begin was British Columbia, which made a political decision in 2001 but did not begin to enact it until about 2003 . They were very organized about it and moved different physicians to centralized centers and added resources to those centers. Alberta was more informal and did not have a formal regionalization process, but did move surgeons into centralized locations. Ontario, with the largest population, has had the biggest effect. About 2004, Ontario started the process, which is still ongoing. That is probably why we see the largest change in volume at the tail end because of the study period.

With regard to the numbers of lobectomies being performed, certainly the incidence of lung cancer is cresting in Canada and it only goes up approximately $1 \%$ per year, so it wouldn't explain the increase in volume performed. I didn't present the data, but over this time frame, the number of pneumonectomies has decreased significantly from approximately 500 per year to approximately 250 per year. So this would be a potential explanation-surgeons operating on those patients are trying to do more limited resections. Alternatively, patients are being referred to these high-volume centers where they are getting multidisciplinary evaluation and not short circuiting the surgeon and going directly into radiation or medical oncologists. These are potential explanations.

Dr Colson. So was it done as a mandate or a financial incentive if it was a high-volume versus low-volume center?

Dr Finley. Increased funding was involved in almost all the regionalizations. Certainly in Ontario and British Columbia it is and was an incentive-driven process, but in other provinces it was more informally done without additional costs.

Dr Colson. The second question. You have stated that the decrease in IHM during this time period is likely related to the shift of cases from low- to high-volume centers. However, given that there is a $20 \%$ increase in the total number of lobectomies over the course of your study, couldn't it be that the increased percentage of lobectomies done at high-volume centers is also just because there are more cases for everyone, and therefore more mid-level centers now qualify as high-volume centers as they cross the case threshold of 60 ?

Dr Finley. Certainly there is a proportion of patients and facilities that cross that threshold, but the majority of the effect we saw was in the reduction of those low-volume centers going out of the business, the reduction of the number of institutions performing it.

Dr Colson. There is an obvious dichotomy between the dramatic increase in mortality associated with an increasing number of patients being treated in high-volume centers, but there is no decrease in mortality associated with increasing the volume at a specific hospital, correct?

Dr Finley. Yes. 
Dr Colson. Given that the volume alone does not seem to be the answer to everything, does your study reflect that the most important benefit of regionalization may actually be the shifting of more cases to low-mortality hospitals, and thus the overall decrease is reflecting more cases done at these low-mortality hospitals, in essence? Is there any insight that you have gained as to how to make these high-volume centers really low-mortality centers?

Dr Finley. That is a good point, because there are a number of small-volume centers that have excellent results with low mortalities. However, on average the larger-volume hospitals deliver better outcomes. Clearly a lot goes into the mortality of a patient beyond the surgeon's involvement. We are intimately involved in our patients' care, but a number of people become involved in their outcomes, from the nursing ratios on the floor to the level of monitoring the patients are undergoing to the ability of rescue. I think one of the most influential articles I read in the last year was by Amir Ghaferi, in The New England Journal of Medicine, who suggested that the difference between a low-mortality hospital and a high-mortality hospital had nothing to do with complication rates, which were exactly the same. It was the ability of rescue; it was the ability to pull those patients out of the fire once the morbidity occurs. I think that is an area of research that needs to continue and be ongoing, because if you don't have 24-hour intensive care or somebody in-house who is experienced with these patients, then you can miss an opportunity to intervene.

Dr Colson. Thank you.

Dr Thomas Egan (Chapel Hill, NC). I enjoyed your presentation, but some of your conclusions are based on the accuracy of coding. In the United States there is a financial incentive for hospitals to code more, and I am curious to know across the provinces during the time of your study, were there any financial incentives and did you notice an increase in the number of ICD-9 codes over time?

Dr Finley. There certainly was an increase in the Charlson comorbidity index as it relates to the ICD-9 and 10 codes. Hospitals in some provinces are remunerated on the comorbidities of their patients, so there could be a slight trend there. That said, the Canadian Institute of Health information goes back and examines the hospitals on their coding systems and publishes regular reports on the accuracy and validity of those reports, and it has actually been quite reliable. That said, within our data it is internally consistent from year to year, so I don't think there was a drift in that way.

Dr Paul Waters (Greenwich, Conn). My question is a specific question about a patient, John Smith. John Smith goes to a smallvolume hospital, say 10 cases per year, and dies. That hospital now has a $10 \%$ mortality. He goes to a big hospital, bigger center, say 100 cases per year, and dies. That is a $1 \%$ mortality. So it seems that smaller centers are kind of inherently disadvantaged. It is a bit like setting up a transplant program. If you do a small volume the first year and have a mortality, you have a bad set of numbers.
You probably mentioned this in your talk and I zoned out, but I would like to hear your response to that.

Dr Finley. As I said, there are some tremendous small-volume hospitals, and I think all of us worry about how to evaluate institutions in a fair way, and that is why on a population basis we look at all the hospitals lumped together. There are hospitals that do a very good job, and trying to elucidate which hospitals actually have a statistically significant deviation from an acceptable threshold is difficult to do in real life, because a hospital that performs 10 lobectomies per year would require 4 or 5 years of evaluation to know if they actually drifted off of statistically acceptable level. So you are right, in a given year in a given hospital, it is unfair to disadvantage them, but this is a population-based study, so we are looking from 20,000 feet and lumping those groups together.

Dr Waters. So that small hospital you looked at for 5 years, and your threshold for acceptable was $6 \%$, it is going to be an unacceptable center on the basis of those numbers, isn't it, if it has a single mortality per year? It is not an argument.

Dr Todd Demmy (Buffalo, $N Y$ ). This regionalization was coincident to the popularity rise in thoracoscopic lobectomy, which has been shown to have a similar reduction in hospital stay, also a reduction in complications, which for a group who may be getting sicker might have contributed to some of these outcomes. Did you look at the proportions of thoracoscopic lobectomy to open in your groups?

Dr Finley. No, we were unable to do that, but I agree with you that different technologies have an effect here. Minimally invasive thoracic surgery and improvements in intensive care, perioperative evaluation, and better staging all help, so we are picking patients who do better. These could all have influenced the outcome.

Dr Valerie Rusch (New York, NY). The optimum care of these patients is really a team effort, demanding excellent care from the anesthesiologist team and nursing team. Do you have any way of examining that in your analyses and has there been any regionalization effort to develop specific nursing support services or the designation of thoracic anesthesia teams to optimize the care of these patients that might also affect your results?

Dr Finley. It is difficult to evaluate from this study, but certainly different provinces have enacted different systems of care as they have taken on this process. In British Columbia, again, with the concentration of specialized surgeons, there has been specialized nursing and education in those centers. In Ontario, again with the concentration of care and incentives to the hospital, those other vital services are strengthened. There are guidelines that were published in The Annals of Thoracic Surgery with recommendations for a single-payer system, and they listed a number of criteria that would be useful for those regionalized centers, including 24-hour interventional radiology, intensive care, and ancillary staff such as specialized nursing and respiratory therapy. But I agree with you that specialized nursing and specialized anesthesia go a long way to improving that care. 